\title{
New role of human ribosomal protein S3: Regulation of cell cycle via phosphorylation by cyclin-dependent kinase 2
}

\author{
SE HEE HAN ${ }^{1}$, JI HYUNG CHUNG ${ }^{2}$, JOON KIM ${ }^{3}$, KEY-SUN KIM ${ }^{1}$ and YE SUN HAN ${ }^{4}$ \\ ${ }^{1}$ Center for Diagnosis, Treatment and Care System of Dementia, Korea Institute of Science and Technology, \\ Seoul 02792; ${ }^{2}$ Department of Applied Bioscience, College of Life Science, CHA University, Pocheon 11160; \\ ${ }^{3}$ Laboratory of Biochemistry, Division of Life Sciences and Biotechnology, Korea University, Seoul 02841; \\ ${ }^{4}$ Department of Advanced Technology Fusion, Konkuk University, Seoul 05029, Republic of Korea
}

Received October 29, 2016; Accepted March 14, 2017

DOI: $10.3892 / \mathrm{ol} .2017 .5906$

\begin{abstract}
Human ribosomal protein S3 (hRpS3) is a component of the $40 \mathrm{~S}$ ribosomal subunit that associated in protein synthesis. hRpS3 has additional ribosomal functions such as DNA repair, transcription, metastasis, and apoptosis via interaction with numerous signaling molecules and has different modifications. Cyclin-dependent kinases (CDKs) are heterodimeric serine/threonine protein kinases that regulate cell cycle progression. Among its members, the Cdk1-cyclin B complex is known to control cell progression in the $\mathrm{G} 2 / \mathrm{M}$ phase, while Cdk2-cyclin E/A complexes function in G1/S and $\mathrm{S} / \mathrm{G} 2$ transition. In our previous study, we observed interaction between $\mathrm{hRpS} 3$ and $\mathrm{Cdk} 1$. The present study investigated the interaction between $\mathrm{hRpS} 3$ and $\mathrm{Cdk} 2$. Cdk2 phosphorylated hRps3 at amino acid residues S6 and T221 during the S-phase. Furthermore, hRpS3 knockdown delayed cell cycle progression by modulating the expression of cell cycle-related proteins, including cyclin B1 and cyclin E1. These findings suggest that hRpS3 is involved in Cdk2-mediated cell cycle regulation.
\end{abstract}

\section{Introduction}

Cyclin-dependent kinase $(\mathrm{Cdk}) 2$ regulates the initiation and progression of the $\mathrm{S}$ phase of the cell cycle, and the regulation of Cdk2 involves cyclin binding and phosphorylation (1).

Correspondence to: Professor Ye Sun Han, Department of Advanced Technology Fusion, Konkuk University, 120 Neungdong-ro, Gwangjin-gu, Seoul 05029, Republic of Korea E-mail: yshan@konkuk.ac.kr

Abbreviations: hRpS3, human ribosomal protein S3; CDK, cyclin-dependent kinase; Aph, aphidicolin; GFP, green fluorescent protein; p-P53, phospho-P53; p-Cdk2, phosphor-Cdk2; NLS, nucleotide localization signal; KH, K homologue domain; IP, immunoprecipitation

Key words: human ribosomal protein S3, cyclin-dependent kinase 2, cell cycle regulation, in vitro kinase assay, protein-protein interaction
Monomeric $\mathrm{Cdk} 2$ is inactive, and its activation requires binding to cyclin. Cdk2 associates with cyclin $\mathrm{E}$ to drive $\mathrm{G} 1 / \mathrm{S}$ phase transition, and it associates with A-type cyclins to mediate DNA replication during $\mathrm{S}$ phase (2-5). The $\mathrm{Cdk} 2 /$ cyclin complex is recognized by multiple kinases, which phosphorylate the T14, Y15, and T160 amino acid residues in the complex. Phosphorylation of T14 and Y15 induces inactivation of Cdk2; activation of $\mathrm{Cdk} 2$ requires de-phosphorylation of both T14 and Y15 by Cdc25 and phosphorylation of T160 by CDK activating kinase (CAK) $(6,7)$. Cdk2 also mediates the cell cycle inhibitory and tumor-suppressing activities of P21 Cip1 and P27 Kip1, respectively (2,8-10).

Human ribosomal protein $\mathrm{S} 3(\mathrm{hRpS} 3)$ is a component of the small ribosomal subunit, which is involved in protein synthesis (11-13). The functions of hRpS3 are not limited to protein translation, and it performs multiple extra-ribosomal activities, such as in DNA repair, cell death, inflammation, and tumorigenesis (13-19). These extra-ribosomal functions of hRpS3 are induced by its interactions many signaling molecules, which result in different post-translation modifications that confer different abilities to hRpS3 (13-19). hRpS3 is phosphorylated by IKK $\beta$ on Serine 209, which is important for nuclear localization (7). In response to DNA damage, hRpS3, phosphorylated by protein kinase $\mathrm{C}-\delta(\mathrm{PKC} \delta)$ at serine 6 (S6) and Threonine 221 (T221) residues, translocates into the nucleus for repair $(11,12,20)$. Moreover, $\mathrm{hRpS} 3$ is also phosphorylated by extracellular signal-regulated kinase 1 (ERK1), which is a MAP kinase that plays an important role in the regulation of cell growth $(11,21,22)$. ERK1 phosphorylates hRpS3 on Threonine 42 (T42) $(11,22)$; moreover, this phosphorylation is necessary for the nuclear translocation of $\mathrm{hRpS} 3$ in response to DNA damage (21). Threonine 70 (T70) of hRpS3 is phosphorylated by Akt, which promotes its nuclear translocation, thereby preventing hRpS3-induced apoptosis by inhibiting its interaction with E2F1 $(13,20)$.

In this study, we showed that Cdk2 also interacts with hRpS3. Moreover, using bioinformatics tools (NetPhos2.0, www.cds.dtu.dk/services/NetPhos; KinasePhos, kinasephos.mbc.nctu.edu.tw), we found eight sites for putative Cdk2-mediated phosphorylation in hRpS3. We also found that Cdk2 phosphorylates S6 and T221 of hRpS3. Cdk2 is active in $\mathrm{S}$ phase, and our in vitro kinase assay revealed that 
hRpS3 phosphorylation in cells arrested in the S-phase was more than two folds that in asynchronous control cells. Cdk2 phosphorylates the non-ribosomal form of $\mathrm{hRpS} 3$, and this phosphorylation is important for nuclear transport of hRpS3. We also observed that knockdown of hRpS3 delays cell cycle progression. Our findings demonstrate that hRpS3 is involved in cell cycle regulation and that this function may be linked to DNA damage repair.

\section{Materials and methods}

Cell lines. Human embryonic kidney cells (HEK293) were grown in Dulbecco's Modified Eagle's medium (DMEM; Invitrogen Life Technologies, Carlsbad, CA, USA) containing $10 \%$ fetal bovine serum (FBS, Invitrogen Life Technologies) and $1 \%$ penicillin-streptomycin solution (Sigma-Aldrich, St. Louis, $\mathrm{MO}, \mathrm{USA}$ ) at $37^{\circ} \mathrm{C}$ in a $5 \% \mathrm{CO}_{2}$ incubator.

Plasmid construction. Gene fragments corresponding to cDNA coding regions of human RpS3 (Accession no. NM_001005) and Cdk2 (accession no. NM_001798) were amplified using PCR. A Cdk2 fragment was inserted into the EcoRI and SalI sites of pCMV Tag2C (Stratagene, La Jolla, CA, USA), and hRpS3 fragments were inserted into the BamHI and XhoI sites of pCMV Tag3A (Stratagene). All constructs were confirmed by restriction enzyme mapping and DNA sequencing.

pDNA transfection. HEK293 cells were seeded in 6-well plates at a density of $1 \times 10^{5}$ cells per well and incubated for $24 \mathrm{~h}$ before transfection. Recombinant plasmid DNAs were transiently transfected into $80 \%$ confluent HEK293 cells using Lipofectamine ${ }^{\circledR} 2000$ reagent (Invitrogen Life Technologies) according to the manufacturer's protocol. After $24 \mathrm{~h}$ of incubation with the plasmids, the cells were collected and lysed in lysis buffer (50 mM Tris- $\mathrm{HCl}, \mathrm{pH} 8.0 ; 100 \mathrm{mM} \mathrm{NaCl} ; 5 \mathrm{mM}$ EDTA; 1 mM NaF; 1 mM Na$V_{4} V_{4} ; 1 \%$ Nonidet P-40; $10 \mu \mathrm{g} / \mathrm{ml}$ of PMSF; and protease inhibitor cocktail) for $40 \mathrm{~min}$ at $4^{\circ} \mathrm{C}$. After centrifugation, supernatants were collected and used in immunoblot analysis and immunoprecipitation (IP).

GST pull-down assay. For GST pull-down assays, GST-Cdk2 was adsorbed onto glutathione-Sepharose 4B beads

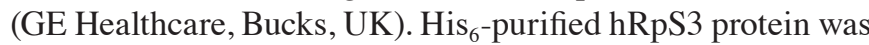
then incubated with GST or GST fusion protein in binding buffer $(50 \mathrm{mM}$ Tris- $\mathrm{HCl}$, pH 7.5; $150 \mathrm{mM} \mathrm{NaCl} ; 1 \mathrm{mM}$ EDTA; $0.3 \mathrm{mM}$ DTT; $0.1 \% \mathrm{NP}-40$; and protease inhibitor cocktail). The binding reaction was performed for $3 \mathrm{~h}$ at $4^{\circ} \mathrm{C}$; subsequently, the beads were washed and bound proteins were subjected to SDS-PAGE analysis. Proteins were detected by immunoblot analysis.

Co-IP. HEK 293 cells were seeded $5 \times 10^{5}$ cells $/ 60-\mathrm{mm}^{2}$ dish and cultured $80-90 \%$ confluency before transfection. Lysates were collected from cells transiently transfected with different sets of plasmid DNAs and incubated for $3 \mathrm{~h}$ with described antibodies at $4^{\circ} \mathrm{C}$ with gentle rotation. Antibodies and bound proteins were incubated with protein A/G-Sepharose beads (Santa Cruz Biotechnology, Inc., Santa Cruz, CA, USA) at $4^{\circ} \mathrm{C}$ for $12 \mathrm{~h}$. Samples were precipitated with centrifugation, washed with PBS, and mixed with gel loading buffer.
Immunoprecipitated samples were resolved on SDS-PAGE gel and subjected to immunoblot analysis.

Immunoblot analysis. Proteins resolved on 8-12\% SDS-PAGE gel were transferred onto polyvinylidene difluoride (PVDF) membranes (Pall Corporation, East Hill, NY, USA). The membranes were blocked with 5\% non-fat dried milk in TBS-T (TBS with $0.05 \%$ Tween-20) for $20 \mathrm{~min}$ at room temperature. The membrane was incubated with primary antibodies against anti-hRpS3, p-Ser hRpS3, p-Thr hRpS3 (Cell Signaling Technology Inc., MA, USA), anti-Cdk2, Cdk1, cyclin B1, cyclin E1 (Santa Cruz Biotechnology). After appropriate washing, the membranes were incubated with horseradish peroxidase (HRP)-conjugated secondary antibodies (Santa Cruz Biotechnology). Protein bands were detected using ECL Western blotting detection reagents (Thermo Fisher Scientific, Waltham, MA, USA).

Cell cycle synchronization. To arrest cells the in the S-phase, cells transfected for $24 \mathrm{~h}$ were treated with $3 \mathrm{mM}$ thymidine (an inhibitor of DNA synthesis) for $18 \mathrm{~h}(23,24)$. Then, the cells were washed with medium and incubated in fresh medium for $2 \mathrm{~h}$. To collect G2/M phase cells, the cells were treated with $0.1 \mu \mathrm{g} / \mathrm{ml}$ nocodazole (a mitotic inhibitor) for $12 \mathrm{~h}(23,24)$. An aliquot of cells was stained with propidium iodide (PI; Sigma-Aldrich) and analyzed by fluorescenceactivated cell sorting (FACS).

FACS analysis. Cells were trypsinized and centrifuged for $10 \mathrm{~min}$. The collected cells were fixed with $70 \%$ ethanol for $30 \mathrm{~min}$, followed by centrifugation. After washing with ice-cold PBS, the cell pellets were resuspended in $0.5 \mathrm{ml}$ PBS containing $50 \mu \mathrm{g} / \mathrm{ml}$ PI and $100 \mu \mathrm{g} / \mathrm{ml}$ RNase (Sigma-Aldrich). After $30 \mathrm{~min}$ of incubation in the dark, cell complex was estimated using a minimum of 10,000 cells per sample and was analyzed using a flow cytometer. Fluorescence emitted from the PI-DNA was analyzed using Cell Quest Alias software (BD Biosciences, Rockville, MD, USA).

In vitro Cdk2 kinase assay. After incubation of cell lysates with Cdk2-specific monoclonal antibody (5 $\mu \mathrm{g})$, samples were incubated with $50 \mu \mathrm{l}$ protein $\mathrm{A} / \mathrm{G}$-Sepharose beads at $4^{\circ} \mathrm{C}$ for $12 \mathrm{~h}$. Immune complexes were washed with washing buffer (1:1 mixture of lysis buffer and PBS) and kinase reaction buffer (20 mM Tris-HCl, $\mathrm{pH} 7.4 ; 15 \mathrm{mM} \mathrm{MgCl}_{2}$; and $1 \mathrm{mM}$ DTT). After washing, samples were added to a mixture of $1 \mu \mathrm{g}$ substrate (histone $\mathrm{H} 1$ or hRpS3), $200 \mu \mathrm{M}$ ATP, and $5 \mu \mathrm{Ci}\left[{ }^{32} \mathrm{P}-\gamma\right]$ ATP in $30 \mu \mathrm{l}$ of reaction buffer. Reactions were carried out for $25 \mathrm{~min}$ at $30^{\circ} \mathrm{C}$ and terminated by addition of SDS-PAGE sample buffer. Samples were subjected to SDS-PAGE, and phosphorylation was detected by autoradiography.

hRpS3 siRNA. hRpS3 siRNA oligonucleotides targeting the sequences 5'-GGGUUCCUAGUACUGCAATT-3' and 5'-UUGCAGUACUAGGAACCCCTT-3' were purchased from Santa Cruz Biotechnology. Cells were transfected with hRpS3 siRNAs (5 nM each) using Lipofectamine ${ }^{\circledR} 2000$ (Invitrogen Life Technologies). Following $24 \mathrm{~h}$ of transfection, the level of hRpS3 was determined using anti-hRpS3 antibody 
A



B

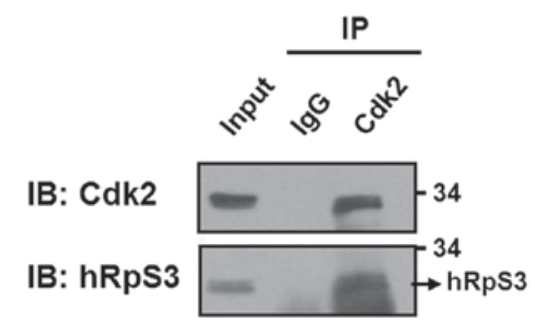

C



Figure 1. Cdk2 interacts with hRpS3 in vitro and in vivo. (A) HEK293 cells were transfected with c-Myc-hRpS3 and empty vector, FLAG-Cdk2, or FLAG-Cdk2-T160A. Whole cell lysates were collected and immunoprecipitated with anti-FLAG antibody and protein A/G Sepharose beads. Immunoblot analysis was performed using anti-hRpS3 and anti-FLAG antibodies. (B) HEK293 cell lysates were immunoprecipitated with anti-Cdk2 antibody, and immunoblot analysis was performed with anti-Cdk2 and anti-hRpS3 antibodies. Rabbit immunoglobulin G (IgG) was used as a negative control. (C) Pull down of purified His-hRpS3 with GST-Cdk2 bound to glutathione sepharose beads. Lane 1 contains purified His-hRpS3 only (input). Lanes 2 and 3 are the pull-down pellets from GST and GST-Cdk2, respectively.

(Cell Signaling Technology Inc., Danvers, MA, USA). A scrambled siRNA [green fluorescent protein (GFP) siRNA, 5'-GGGCACAAGCUGGAGUACAAC UAC A-3'] was used as the control.

Statistical analysis. All experiments were performed at least three times for statistical analysis. To determine the significance of the differences between samples, we performed the Student's t-test using Microsoft Excel. Date were expressed as mean \pm standard errors, and $\mathrm{P}<0.05$ (paired two-tailed t-test, $\mathrm{P}<0.01, \mathrm{P}<0.001)$ was considered to indicate statistical significance.

\section{Results}

Cdk2 physically interacts with hRpS3. To determine whether hRpS3 interacts with Cdk2, co-IP was conducted using HEK293 cells transfected with FLAG empty vector or with FLAG-tagged Cdk2 (FLAG-Cdk2) and c-Myc-tagged hRpS3. After $24 \mathrm{~h}$ incubation, cells were lysed and immunoprecipitated with anti-RpS3 antibody. The co-IP results indicated that Cdk2 interacted with ectopically expressed hRpS3 (Fig. 1A). A similar co-IP assay with antibodies against endogenous Cdk2 and $\mathrm{hRpS} 3$ also revealed that $\mathrm{Cdk} 2$ interacted with $\mathrm{hRpS} 3$ (Fig. 1B). Rabbit IgG was used as a control. To determine whether hRpS3 interacts directly with Cdk2, we conducted a GST pull-down assay (Fig. 1C). His-hRpS3 and GST-Cdk2 were generated and purified before conducting the assay.
Purified His-hRpS3 interacted with GST-Cdk2 but not with GST alone, indicating that Cdk2 interacts directly with hRpS3. Next, we investigated whether the interaction between Cdk2 and $\mathrm{hRpS} 3$ required $\mathrm{Cdk} 2$ activation. For this, we prepared FLAG-Cdk2 and a mutant of Cdk2 with modifications at T160 (T160A; Cdk2 phosphorylated at T160 is the active form), and transfected these into HEK293 cells (Fig. 1A; lanes 2 and 3). Co-IP revealed that substitution of threonine with alanine inhibited Cdk2 phosphorylation at the site. These results show that hRpS3 interacts only with the active form of Cdk2 (Fig. 1A; lanes 2 and 3).

hRpS3 was phosphorylated by Cdk2 during the S-phase. It is already known that the highest activity of Cdk 2 is observed in the S-phase (1). Therefore, we investigated the association between $\mathrm{hRpS} 3$ phosphorylation and Cdk2 activity. Co-IP of asynchronous and synchronous cells arrested at the $\mathrm{S}$ and G2/M phases was conducted using anti-hRpS3 antibody (Fig. 2A). Consistent with the results in Fig. 1A, endogenous hRpS3 was found to interact with Cdk2. Moreover, Fig. 2A shows that this interaction was higher in the S-phase than in the $\mathrm{G} 2 / \mathrm{M}$ phase. This result indicates that $\mathrm{hRpS} 3$ interacts with the active form of Cdk2 in the S-phase.

Cdk2 phosphorylates its substrate at the serine/threonine residues. Therefore, the possibility of $\mathrm{hRpS} 3$ being a substrate of Cdk2 was examined. We have previously demonstrated that Cdk1 interacts and phosphorylates hRpS3, and this phosphorylation increased in the G2/M phase (21). Thus, we conducted a 
A

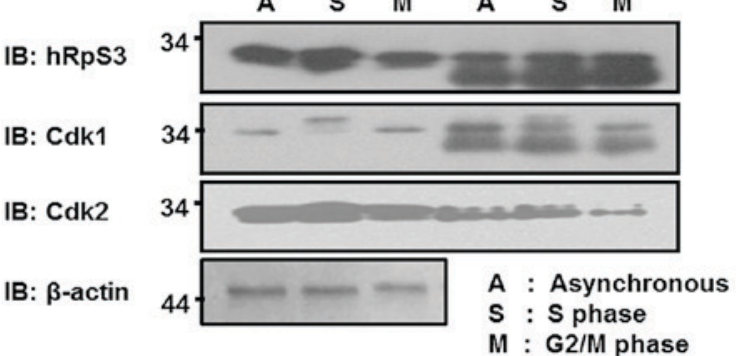

B

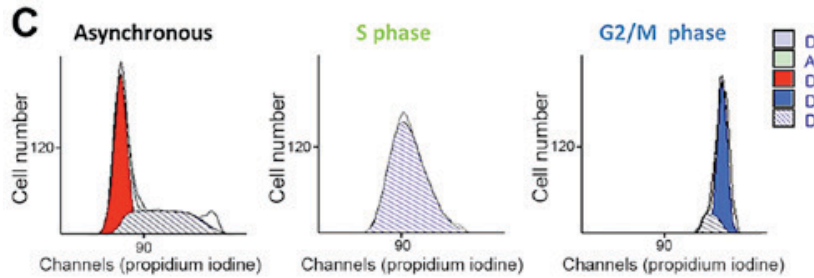

Histion H1
His-hRpS3
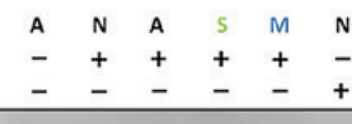

A S M

Cdk2 assay



D

D

IB: CdkI
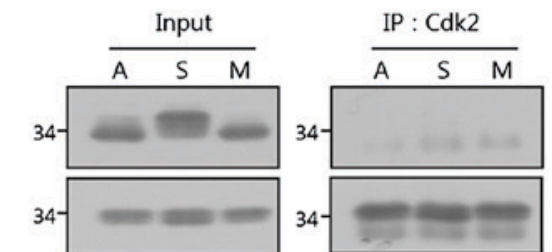

IB: Cdk2

IB: Cdk2

p-160

IB: Cyclin E1

IB: Cyclin B1

IB: $\beta$-actin



A: Asynchronous

S: S phase

$\mathrm{M}$ : $\mathrm{G} 2 / \mathrm{M}$ phase

$\mathrm{N}$ : No Beads A: Asynchronous $5:$ s phase $M: G 2 / M$ phase

Figure 2. hRpS3 phosphorylation by Cdk2, mainly in S phase. (A) HEK293 cells were arrested in various phases of the cell cycle by thymidine (S phase) and nocodazole (G2/M phase). Whole cell lysates were immunoprecipitated with anti-hRpS3 antibody and were immunoblotted using anti-Cdk1/2 and anti-hRpS3 antibodies. (B) Samples treated similar to those in (A) were subjected to immunoprecipitation with anti-hRpS3 antibody. Immunoblot analysis was conducted with anti-hRpS3, anti-p-Ser, and anti-p-Thr antibodies. (C) The population of asynchronous or synchronous cells in different cell cycle phases was analyzed using FACS. Cdk2 was isolated from asynchronous or synchronous cells by immunoprecipitation with anti-Cdk2 antibody. The precipitates were used for in vitro kinase assay with histone $\mathrm{H} 1$ and hRpS3 purified protein. (D) Whole cell lysates were prepared and subjected to immunoblot analysis with corresponding antibodies. The specificities of Cdk1/2 antibodies were observed by immunoprecipitation with anti-Cdk2 and immunoblot with anti-Cdk1 and anti-Cdk2. The level of Cdk1, Cdk2, cyclin B1, and cyclin E1 in cell lysates was determined by western blotting using the corresponding antibodies. $\beta$-Actin was used as loading control. The immunoprecipitated sample was used to confirm cross-reaction of antibodies.

co-IP assay using asynchronous and synchronous cells (arrested at the $\mathrm{S}$ and $\mathrm{G} 2 / \mathrm{M}$ phases). Whole cell lysates were subjected to immunoprecipitation with anti-hRpS3 antibody. Following immunoblot analysis using anti-hRpS3, hRpS3 was detected in both input and immunoprecipitation samples (Fig. 2B). Phospho-serine ( $\mathrm{p}$-Ser) levels in the immunoprecipitated sample were higher in the cells arrested at the S-phase than in the cells arrested at the $\mathrm{G} 2 / \mathrm{M}$ phase. In contrast, an intense band of phospho-threonine (p-Thr) was detected in cells in the G2/M phase. Since Cdk2 is active in the S-phase, these results indicate that $\mathrm{Cdk} 2$ phosphorylates the serine of hRpS3 in the S-phase.

Fig. 1. A shows that hRpS3 binds with the active form of Cdk2. To demonstrate the ability of $\mathrm{Cdk} 2$ to phosphorylate $\mathrm{hRpS} 3$, we conducted an in vitro kinase assay (Fig. 2C). Cells were synchronized at the G2/M- and S-phases using nocodazole and thymidine, respectively. Cell synchronization was confirmed by FACS analysis (Fig. 2C, upper histogram). The in vitro kinase assay was conducted using hRpS3 and histone H1. Cdk2 was isolated from the synchronized samples by immunoprecipitation with anti-Cdk2 antibody. histone $\mathrm{H} 1$, a known substrate of $\mathrm{Cdk} 2$, was used as a positive control. Autoradiography revealed phosphorylation of both histone H1 and hRpS3 by Cdk2. Moreover, phosphorylation of hRpS3 by $\mathrm{Cdk} 2$ increased in the S-phase.

$\mathrm{Cdk} 1$ and $\mathrm{Cdk} 2$ are closely related proteins with high similarity. Thus, there might be a cross-reaction among the antibodies used for co-IP. To exclude this possibility, we tested the specificity of anti-Cdk1 and anti-Cdk2 antibodies by immunoprecipitation. The first panel in Fig. 2D shows that the antibody against $\mathrm{Cdk} 1$ specifically recognized $\mathrm{Cdk} 1$ in input, but it did not detect $\mathrm{Cdk} 1$ or $\mathrm{Cdk} 2$ in the immunoprecipitation assay. The same result was obtained for the anti-Cdk2 antibody (Fig. 2D, second panel). Thus, the result for the in vitro $\mathrm{Cdk} 1 / 2$ kinase assay was specific for the indicated protein.

Cdk2 phosphorylates hRpS3 at specific residues. Based on the analysis of $\mathrm{hRpS} 3$ amino acid sequence using bioinformatics tools (NetPhos2.0, www.cds.dtu.dk/services/NetPhos; KinasePhos, kinasephos.mbc.nctu.edu.tw), eight possible sites for phosphorylation by Cdk2 were identified (Fig. 3A) (18). To observe which amino acid is phosphorylated by Cdk2, hRpS3 constructs containing a serine/threonine-to-alanine substitution were created and used as the substrate in the in vitro kinase assay. As shown in Fig. 3B, phosphorylation was significantly 
A


Figure 3. S6 and T221 of hRpS3 were phosphorylated by Cdk1/2. (A) The amino acid sequence of $\mathrm{hRpS} 3$. hRpS3 has 8 possible phosphorylation sites (red). NLS, nuclear localization signal (yellow, amino acid 7-10); $\mathrm{KH}, \mathrm{K}$ homologue domain (blue, amino acids 42-111). (B) Autoradiograph from kinase assays carried out using Cdk1/2 immunoprecipitated lysates of HEK293 cells expressing wild type or various point mutants of hRpS3. Equal amounts of samples were analyzed by coomassie staining.

lower in the hRpS3-S6A and -T221A mutants than in the wild type. This result suggests that Cdk2 phosphorylates the S6 and T221 residues of hRpS3. Coomassie blue staining in the lowest panel was used as a loading control.

hRpS3 knockdown induced phosphorylation of Cdk2 and $P 53$. The physiological effect of interaction between Cdk2 and hRpS3 on the cell cycle was observed by knockdown of hRpS3. For hRpS3 knockdown, HEK293 cells were transfected with varying concentrations siRpS3. Cells transfected with siGFP were used as control. As shown in Fig. 4A, hRpS3 knockdown was successful in all transfected cells. Whole cell lysates were subjected to immunoblotting for the detection of phosphor-Cdk2 (p-Cdk2) (T14, Y15; inactive form), phospho-P53 (p-P53), and P53. The expression of p-Cdk2 (T14, Y15) and p-P53 increased with increasing concentration of siRpS3. Phosphorylation of P53 induced production of p21, an inhibitor of Cdk2 activation (9). Therefore, increased phosphorylation of P53 and Cdk2 (T14, Y15) is indicative of delayed cell cycle progression. This result suggests that $\mathrm{hRpS} 3$ is involved in cell cycle regulation by inducing phosphorylation of P53.

Cell cycle delay was induced by knockdown of hRpS3. The effect of hRpS3 knockdown was examined in synchronous and asynchronous cells by FACS and immunoblotting. To synchronize cells at the late G1 phase, they were treated with aphidicolin (Aph). After $15 \mathrm{~h}$, Aph was released into fresh medium, and cells were harvested at different release times such that they would synchronize and enter the S-phase in 2-4 h (22). Cells enter the G2-phase after $8 \mathrm{~h}$ of releasement.

We observed a slight difference in cell cycle progression in siRpS3-transfected cells and siGFP-transfected control cells
(Fig. 4B). In the hRpS3 knockdown cells, the S and G2/M phases were delayed compared with that in siGFP-transfected control cells. siGFP-transfected cells entered the S-phase at 0-3 $\mathrm{h}$ and the G2/M phase at $5 \mathrm{~h}$ after release of Aph into fresh media. In contrast, the siRpS3-transfected cells were still in the G1-phase at 0-3 h, and entered the G2/M phase at $5 \mathrm{~h}$ after Aph release. Notably, at $5 \mathrm{~h}$ after Aph release, the number of siGFP-transfected cells that entered the G2/M phase was considerably higher than that of siRpS3-transfected cells. Thus, the hRpS3 knockdown cells showed delayed cell cycle progression in each phase compared with the siGFP-transfected control cells. These results suggest that hRpS3 is required for cell cycle progression.

Further, to elucidate the mechanism through which $\mathrm{hRpS} 3$ regulates cell cycle progression, we examined the expression of cell cycle-related proteins using immunoblotting (Fig. 4C). In the siGFP-transfected cells, the level of cyclin B1 (binds to Cdk1 and involved in regulating G2/M transition) significantly decreased after $10 \mathrm{~h}$ of Aph releasement (Fig. 4C). Additionally, in hRpS3 knockdown cells, the level of cyclin B1 significantly decreased after release for $12 \mathrm{~h}$. These results indicate that hRpS3 knockdown induced delay of G2/M progression. The level of cyclin E1 (binds to $\mathrm{Cdk} 2$ and required for progression to the $\mathrm{S}$-phase) in siGFP-transfected and siRpS3-transfected cells decreased after $8 \mathrm{~h}$ of releasement. The level of cyclin E1 in siGFP-transfected cells increased significantly after $12 \mathrm{~h}$ releasement, whereas it was not detected in siRpS3-transfected cells after $8 \mathrm{~h}$ of release. This expression pattern of cyclin E1 showed that hRpS3 knockdown delayed the progression to S-phase. These findings suggest that hRpS3 is regulates cell cycle progression modulating the expression of cell cycle-related proteins (cyclins B and E1).

Cell cycle progression is mainly regulated by CDKs. CDK activity increased or decreased as the cell cycle progressed. Among the CDKs, the Cdk1/cyclin B complex is known to control G2/M transition, while Cdk2/cyclin E1/A complexes regulate $\mathrm{G} 1 / \mathrm{S}$ and $\mathrm{S} / \mathrm{G} 2$ transitions $(9-11,21,25)$. We found that the levels of $\mathrm{Cdk} 2 /$ cyclin $\mathrm{E} 1$ and $\mathrm{Cdk} 1 /$ cyclin B1 were decreased in hRpS3 knockdown cells. Thus, hRpS3 knockdown delayed cell cycle progression by downregulating the proteins involved in cell cycle regulation.

\section{Discussion}

The cell cycle is regulated by the CDK family of proteins (26). CDKs are the catalytic subunits of a large family of heterodimeric serine/threonine protein kinases that regulate cell cycle progression (27). The catalytic activity of CDKs requires the binding of a regulatory subunit, cyclin, which is synthesized and degraded during each cycle (28). The catalytic activity of $\mathrm{Cdk} 1$ requires the binding of the cyclin B1 (27). When Cdk1/cyclin B1 activity is maximal during G2-M phase, this complex phosphorylates a number of proteins that regulate several cellular events (29). Cdk2 plays a role in G1/S transition and initiation of DNA synthesis in the S-phase by forming complexes with cyclins $\mathrm{E}$ and $\mathrm{A}(1,5-7)$.

DNA damage repair and cell cycle progression are tightly regulated. As a ribosomal protein, $\mathrm{hRpS} 3$, is also known to 
A

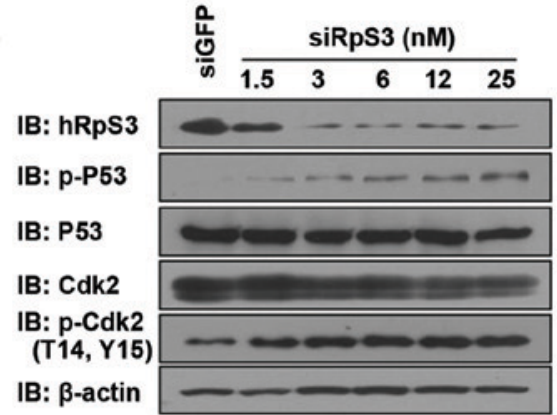

C


IB: $\beta$-actin $43-\infty-\infty-\infty-\infty-\infty-\infty-\infty-\infty-\infty$
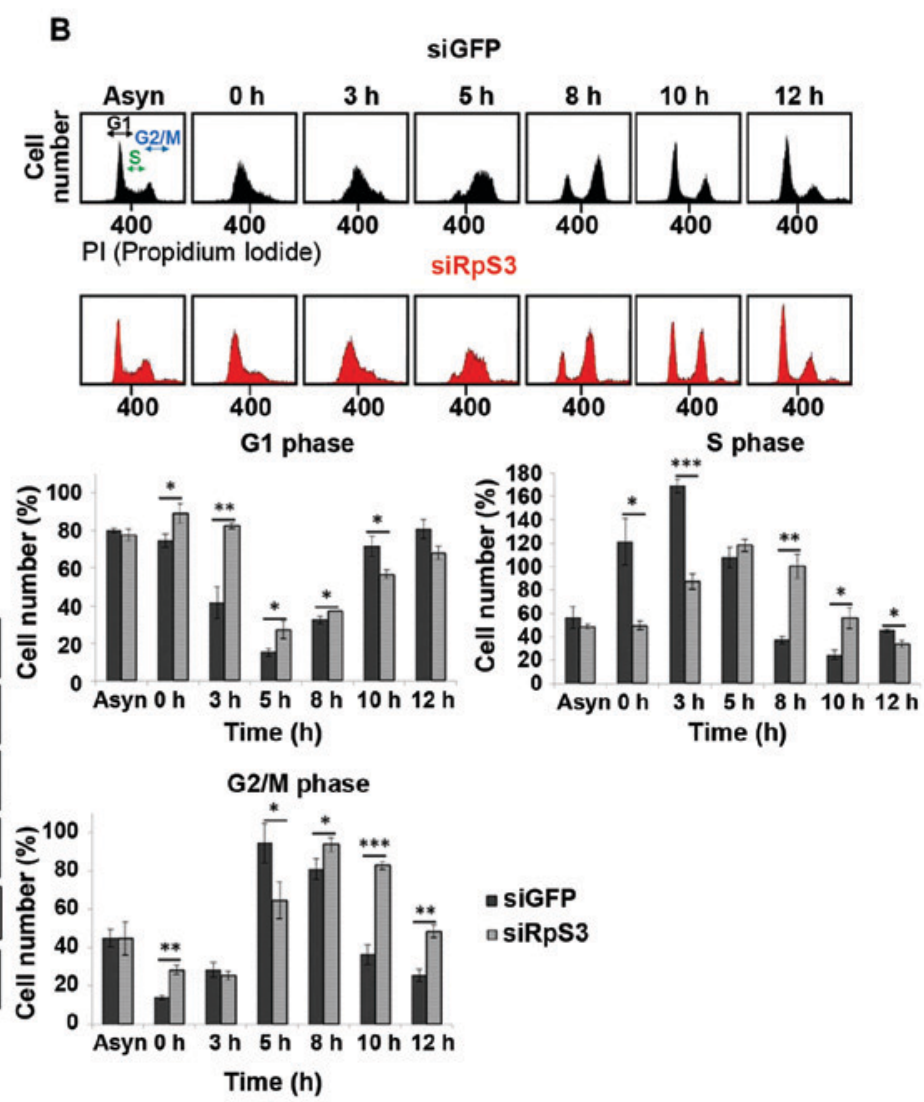

Figure 4. Cell cycle delay induced by knockdown of hRpS3. (A) hRpS3 knockdown induces phosphorylation of Cdk2 and P53. Cells were either treated with siGFP as a control or with various concentrations of siRpS3 (1.5, 3, 6, 12 and $25 \mathrm{nM})$. Whole cell lysates were subjected to immunoblotting with antibodies against hRpS3, Cdk2, p-Cdk2 (T14, Y15), p-P53. $\beta$-Actin was used as loading control. (B and C) HEK293 cells were transfected with siGFP (control) or siRpS3. Cells were arrested in the G1 phase by incubation with aphidicoline for $15 \mathrm{~h}$, released into fresh medium, and harvested at indicated times. (B) Each harvested sample was subjected to flow cytometry. Harvested cells were stained with propidium iodide for FACS analysis. The percentage of siGFP- and siRpS3-treated cells in the indicated cell cycle phase is shown as mean \pm standard error (of three independent experiments). P-value was calculated using a paired t-test, ${ }^{*} \mathrm{P}<0.05,{ }^{* *} \mathrm{P}<0.01,{ }^{* * *} \mathrm{P}<0.001$. (C) Lysates were immunoblotted with antibodies against Cdk1, Cdk2, cyclin B1, cyclin E1, and hRpS3.

perform other extra-ribosomal functions, such as in DNA repair, via its $\mathrm{N}$-glycosylase activity and by releasing 8 -oxoG from a DNA lesion $(30,31)$. Furthermore, hRpS3 binds and stimulates the activity of uracil-DNA glycosylase (UNG), as well as the base excision repair (BER) enzymes, hOGG1 and APE/Ref-1 $(32,33)$. Previous reports have shown that $\mathrm{hRpS} 3$ can be translocated into the nucleus for regulation of cell cycle progression, similar to some other ribosomal proteins such as ribosomal protein L6 and ribosomal protein S13, which are involved in the regulation of cyclin $\mathrm{E}$ and the promotion of cell growth $(34,35)$. Therefore, different functions of $\mathrm{hRpS} 3$ are believed to result from the post-translational modifications that result from its interaction with different molecules.

Previously, we reported that hRpS3 physically interacts with and is phosphorylated by Cdk1, especially, in the G2/M phase. This phosphorylation occurs at T221 and is important for the nuclear translocation of hRpS3 (21). Here, we studied the interaction between $\mathrm{hRpS} 3$ and Cdk2 (Fig. 1). Interestingly, the interaction between $\mathrm{hRpS} 3$ and $\mathrm{Cdk} 2$ was increased in the S-phase. Previous observations prompted us to determine whether hRpS3 is a substrate of Cdk2. We conducted a Cdk2 kinase assay in asynchronous and synchronous cells (arrested in the $\mathrm{S}$ and $\mathrm{G} 2 / \mathrm{M}$ phases) using hRpS3 and purified histone $\mathrm{H} 1$ as substrates (Fig. 2C) and observed that hRpS3 phosphorylation by Cdk2 occurs in the S-phase. These results revealed that hRpS3 interacts with $\mathrm{Cdk} 2$ and is phosphorylated at a serine residue by $\mathrm{Cdk} 2$. Subsequently, based on a previous report and the $\mathrm{hRpS} 3$ amino acid sequence analysis performed in this study, eight phosphorylation sites were identified (Fig. 3A). The serine/threonine residues in these putative phosphorylation sites were substituted by alanine and a Cdk1/2 kinase assay was performed (Fig. 3B). S6 and T221 of hRpS3 were phosphorylated by Cdk2, and T221 was phosphorylated by Cdk1.

Knockdown of hRpS3 induced phosphorylation of P53, which functions upstream of $\mathrm{Cdk} 1 / 2$, resulting in a delay in cell cycle progression and cell cycle arrest (Fig. 4A). Furthermore, we observed that knockdown of hRpS3 induced delay in cell cycle progression and downregulation of cyclins E1 and B1 (Fig. 4B and C). Our results show that the hRpS3 participates in cell cycle regulation by modulating the expression of cell cycle-related proteins.

In summary, we have shown that $\mathrm{hRpS} 3$ is involved in the Cdk2-mediated regulation of cell cycle progression. Our findings indicate a possible link between DNA damage repair and cell cycle progression. Further studies are required to determine other phosphorylation sites of $\mathrm{hRpS} 3$ that are linked to cell cycle progression and to elucidate the relationship between the mechanisms involved in DNA damage repair and cell cycle control. 


\section{Acknowledgements}

This study was supported by a grant of the Korea Health Technology R\&D Project through the Korea Health Industry Development Institute (KHIDI), funded by the Ministry of Health \& Welfare, Republic of Korea (grant no. HI15C1540) and the National Research Foundation of Korea (NRF) grant funded by the Korean government (MSIP) (no. NRF-2015R1C1A2A01053623).

\section{References}

1. Gu Y, Rosenblatt J and Morgan DO: Cell cycle regulation of CDK2 activity by phosphorylation of Thr160 and Tyr15. EMBO J 11: 3995-4005, 1992.

2. Aleem E, Kiyokawa H and Kaldis P: Cdc2-cyclin E complexes regulate the G1/S phase transition. Nat Cell Biol 7: 831-836, 2005.

3. Koff A, Giordano A, Desai D, Yamashita K, Harper JW, Elledge S, Nishimoto T, Morgan DO, Franza BR and Roberts JM: Formation and activation of a cyclin E-cdk2 complex during the G1 phase of the human cell cycle. Science 257: 1689-1694, 1992.

4. Dulić V, Lee E and Reed SI: Association of human cyclin E with a periodic G1-S phase protein kinase. Science 257: 1958-1961, 1992.

5. Lew DJ, Dulić V and Reed SI: Isolation of three novel human cyclins by rescue of G1 cyclin (Cln) function in yeast. Cell 66 $1197-1206,1991$.

6. Coulonval K, Bockstaele L, Paternot S and Roger PP: Phosphorylations of cyclin-dependent kinase 2 revisited using two-dimensional gel electrophoresis. J Biol Chem 278: 52052-52060, 2003.

7. Wan F, Weaver A, Gao X, Bern M, Hardwidge PR and Lenardo MJ: IKK $\beta$ phosphorylation regulates RPS3 nuclear translocation and $\mathrm{NF}-\kappa \mathrm{B}$ function during infection with Escherichia coli strain O157:H7. Nat Immunol 12: 335-343, 2011.

8. Morgan DO: Cyclin-dependent kinases: Engines, clocks, and microprocessors. Annu Rev Cell Dev Biol 13: 261-291, 1997.

9. Adams PD, Sellers WR, Sharma SK, Wu AD, Nalin CM and Kaelin WG Jr: Identification of a cyclin-cdk2 recognition motif present in substrates and p21-like cyclin-dependent kinase inhibitors. Mol Cell Biol 16: 6623-6633, 1996.

10. Martin A, Odajima J, Hunt SL, Dubus P, Ortega S, Malumbres M and Barbacid M: Cdk2 is dispensable for cell cycle inhibition and tumor suppression mediated by p27(Kip1) and p21(Cip1). Cancer Cell 7: 591-598, 2005

11. Kim SH, Lee JY and Kim J: Characterization of a wide range base-damage-endonuclease activity of mammalian $\mathrm{rpS} 3$. Biochem Biophys Res Commun 328: 962-967, 2005.

12. Hegde V, Kelley MR, Xu Y, Mian IS and Deutsch WA: Conversion of the bifunctional 8-oxoguanine/beta-delta apurinic/apyrimidinic DNA repair activities of Drosophila ribosomal protein S3 into the human S3 monofunctional beta-elimination catalyst through a single amino acid change. J Biol Chem 276: 27591-27596, 2001.

13. Gao X and Hardwidge PR: Ribosomal protein S3: A multifunctional target of attaching/effacing bacterial pathogens. Front Microbiol 2: 137, 2011.

14. Warner J and McIntosh KB: How common are extraribosomal functions of ribosomal protein? Mol Cell 34: 3-11, 2009.

15. Zimmermann RA: The double life of ribosomal proteins. Cell 15: $130-132,2003$
16. Naora $\mathrm{H}$ : Involvement of ribosomal proteins in regulating cell growth and apoptosis: Translational modulation or recruitment for extraribosomal activity? Immunol Cell Biol 77: 197-205, 1999.

17. Kim J, Chubatsu LS, Admon A, Stahl J, Fellous R and Linn S: Implication of mammalian ribosomal protein $\mathrm{S} 3$ in the processing of DNA damage. J Biol Chem 270: 13620-13629, 1995.

18. Kim TS, Kim HD and Kim J: PKCdelta-dependent functional switch of rpS3 between translation and DNA repair. J Biochim Biophys Acta 1793: 395-405, 1793, 2009.

19. Kim TS, Kim HD, Shin HS and Kim J: Phosphorylation status of nuclear ribosomal protein $\mathrm{S} 3$ is reciprocally regulated by protein kinase C\{delta\} and protein phosphatase 2A. J Biol Chem 284: 21201-21208, 2009.

20. Lee SB, Kwon IS, Park J, Lee KH, Ahn Y, Lee C, Kim J, Choi SY, Cho SW and Ahn JY: Ribosomal protein S3, a new substrate of Akt, serves as a signal mediator between neuronal apoptosis and DNA repair. J Bio Chem 285: 29457-29468, 2010.

21. Yoon IS, Chung JH, Hahm SH, Park MJ, Lee YR, Ko SI, Kang LW, Kim TS, Kim J and Han YS: Ribosomal protein S3 is phosphorylated by $\mathrm{Cdk} 1 / \mathrm{cdc} 2$ during G2/M phase. BMB Rep 8: 529-534, 2011.

22. Spandari S, Sala F and Pedrali-Noy G: Aphidicolin: A specific inhibitor of nuclear DNA replication in eukaryotes. Trends Biochem Sci 7: 29-32, 1982.

23. Harper JV: Synchronization of cell population in G1/S and G2/M phases of the cell cycle. Methods Mol Biol 296: 157-166, 2005.

24. Cude K, Wang Y, Choi HJ, Hsuan SL, Zhang H, Wang CY and Xia Z: Regulation of the G2-M cell cycle progression by the ERK5-NFkappaB signaling pathway. J Cell Biol 177: 253-264, 2007.

25. Watanabe N, Broome M and Hunter T: Regulation of the human WEE1Hu CDK tyrosine 15-kinase during the cell cycle. EMBO J 14: 1878-1891, 1995.

26. Malumbers M and Barbacid M: To cycle or not to cycle: A critical decision in cancer. Nat Rev Cancer 1: 222-231, 2001.

27. Malumbers M and Barbacid M: Mammalian cyclin-dependent kinases. Trends Biochem Sci 30: 630-641, 2005.

28. Ecans T, Rosethal ET, Youngblom J, Distel D and Hunt T: Cyclin: A protein specificed by maternal mRNA in sea urchin eggs that is destroyed at each cleavage division. Cell 33: 389-396, 1983.

29. Salaun P, Rannou Y and Prigent C: Cdk1, Plks, Auroras and Neks: The mitotic bodyguards. Adv Exp Med Biol 617: 41-56, 2008.

30. Yacoub A, Augen L, Kelley MR, Doetsch PW and Deutsch WA: A Drosophila ribosomal protein contains 8-oxoguanine and abasic site DNA repair activities. EMBO J 15: 2306-2312, 1996.

31. Deutsch WA, Yacoub A, Jaruga P, Zastawny TH and Disdaroglu M: Characterization and mechanism of action of Drosophila ribosomal protein S3 DNA glycosylase activity for the removal of oxidatively damage DNA bases. J Biol Chem 272: 32857-32860, 1997.

32. Ko SI, Park JH, Park MJ, Kim J, Kang LW and Han YS: Human ribosomal protein S3 (hRpS3) interacts with uracil-DNA glycosylase (hUNG) and stimulates its glycosylase activity. Mutat Res 648: 54-64, 2008.

33. Hegde V, Wang M and Deutsch WA: Human ribosomal protein $\mathrm{S} 3$ interacts with DNA base excision repair protein hAPE/Ref-1 and hOGG1. Biochemistry 43: 14211-14217, 2004.

34. Bernstein KA and Baserga SJ: The small subunit processome is required for cell cycle progression at G1. Mol Biol Cell 15: 5038-5046, 2004.

35. Guo X, Shi Y, Gou Y, Li J, Han S, Zhang Y, Huo J, Ning X, Sun L, Chen $\mathrm{Y}$, et al: Human ribosomal protein S13 promotes gastric cancer growth through down-regulating p27 (Kip1). J Cell Mol Med 15: 296-306, 2011. 